\title{
Effects of Ultrasound versus Low Level Laser Therapy in Levofloxacin-Induced Rat Model of Knee Osteoarthritis
}

\author{
Ghada S Mahmoud ${ }^{1}$, Mohamed Elbedewey ${ }^{2}$, Ahmed M. Abdelkader ${ }^{3}$, \\ Ahmed B. Bekheet ${ }^{4}$, Dina M. El-Kossy ${ }^{5}$, Samir A. Sabbahi ${ }^{6+}$ \\ ${ }^{I}$ Assistant Professor, Departments of Medical Physiology, Faculty of Medicine, Assiut University, Assiut, Egypt, \\ "Visitor Assistant Professor, Departments of Basic Medical Science, Faculty of Physical Therapy, Deraya \\ University, El-Minya, Egypt. ${ }^{2,3,4}$ Lecturer, Department of Internal Medicine, Faculty of Physical Therapy, \\ Deraya University, MinyaElgadida, Minya, Egypt, Instructor, Faculty of Veterinary Medicine, Department of \\ Basic Medical Science, MinyaElgadida, Minya, Egypt. ${ }^{6}$ Professor, Department of Basic Medical Science, \\ Faculty of Physical Therapy, Deraya University, MinyaElgadida, Minya, Egypt. \\ ${ }^{+}$Dean Faculty of Physical Therapy, Deraya University, MinyaElgadida, Minya, Egypt.
}

\begin{abstract}
:
Objectives. Osteoarthritis $(\mathrm{OA})$ is one of the most common diseases affecting both aged and young people. Physiotherapy provides effective non-pharmacologic intervention for knee OA. Evidence for inflammation in $O A$ is good predictive factor of OA progressions. The purpose of this study is to compare the effects of conventional Ultra Sound (US) and low level laser therapy (LLLT) on levels of inflammatory cytokines and histopathological changes in chemically-induced knee $O A$ in rats.

Methods. Eighteen male rats divided into three groups; (Lev) group treated with levofloxacin (Lev), (US) group treated with Lev plus US and (LLLT) group treated with Lev plus low level laser therapy. All animals received total of $200 \mathrm{mg}$ injection of Lev. Total of ten sessions of US and laser applied.

Results. We found significant decrease of serum IL-1beta, Mankin's score and increase in knee extension angle in both US and LLLT groups compared to Lev group. US significantly lower IL-6, INF-gamma, CRP and significantly elevate IL-10 in US group compared to Lev and LLLT groups.

Conclusion. Based on our finding, US may provide better therapeutic effect compared to LLLT in knee osteoarthritis as indicated by reduction of inflammatory cytokines, elevation of anti-inflammatory cytokines and better histopathological score.

Abbreviations: Osteoarthritis (OA), Levofloxacin (Lev), Ultrasound (US), Low level laser therapy (LLLT), Levofloxacin (Lev), Interleukin 1 beta (IL-1B), Interleukin 6 (IL-6), Interleukin 10 (IL-10), Interferon gamma $(I N F-\gamma), C$-reactive protein $(C R P)$, Tumor necrosis factor alpha $(T N F-\alpha)$.

Key words: Osteoarthritis (OA), Levofloxacin (Lev), Ultrasound (US), Low level laser therapy (LLLT), Interleukin 1 beta (IL-1 $\beta)$, Interleukin 6 (IL-6), Interleukin 10 (IL-10), Interferon gamma (INF- $\gamma), C$-reactive protein (CRP), Tumor necrosis factor alpha $(T N F-\alpha)$.
\end{abstract}

\section{Introduction}

Osteoarthritis (OA) is chronic degenerative joint disease that is considered as the most common musculoskeletal disorders affecting more than 25 million adults in the united states[1]. Evidence for inflammation in OA as a predictive factor of OA progression has been reported by several previous studies. Inflammation is indicated by increased expression of inflammatory markers IL-1 beta, IL-6, IL-15, IL-17, IL-18 andTNF- $\alpha[2]$. Toxic compounds such as quinolones and collagenase have been used to develop chemically induced models of OA. Oral quinolone antibiotics usually cause growth defects in young children. This occurs through their action on the epiphyseal growth plate of their bones. It can also cause loss of proteoglycans and chondrocytes through systemic administration[3]. This mechanism serves the use of this antibiotic in causing lesions in animals, though it does not cause osteophyte formation[4].

Continuous US represents safe and effective method in the management of knee osteoarthritis[5],[6].LLLT has analgesic effect and improves microcirculation in knee OA[7]. The use of LLLT and exercise training program provide better effect than exercise training alone in management of chronic OA of knee joint[8].Although physiotherapy of OA has recently greatly advanced, few studies have evaluated the underlying mechanisms and compare the efficacy of different methods. The purpose of this study is to compare the effects of continuous US and LLLToninflammatory markers and histopathological changes in knee joint usingchemically-induced rat model of OA. 


\section{II.1Drugs and chemicals:}

\section{Material and Methods}

Levofloxacin (Levoxin) $500 \mathrm{mg}$ (AMOUN PHARMACEUTICAL Co.SAE, El-Obour City, Cairo, Egypt). The tablet $500 \mathrm{mg}$ is grounded and dissolved in $25 \mathrm{ml}$ normal saline (2\% solution). Rat CRP (CReactive Protein ELIZA Kit Cat. No: E-EL-R0022); Rat IL-1 $\beta$ (Interleukin 1 Beta ELIZA Kit, Cat. No: E-ELR0012); Rat IL-6 (Interleukin 6 ELIZA Kit, Cat No.: E-EL-R0015); Rat IL-10 (Interleukin 10 ELIZA Kit, Cat. No: E-EL-R0016); Rat INF- $\gamma$ (Interferon Gamma ELIZA Kit, Cat. No: E-EL-R0009);Rat TNF- $\alpha$ (Tumor Necrosis Factor Alpha ELIZA Kit, Cat. No: E-EL-R0019). Rat ELIZA Kits for detecting CRP, IL-6, IL-1 $\beta$, IL10, TNF- $\alpha$, and INF- $\gamma$ purchased from Elabscience Biotechnology Co.,Ltd, Building 4, Room 403, Guandong Science and Technology Industry Park,WuHan,P.R.C., China.

\section{II.2 Equipment:}

Stat Fax ${ }^{\circledR 2} 100$ - Microplate Reader (Awareness Technology, Inc. PO Drawer 1679, Palm City, USA, FL 34991. OLYMPUS Laboratory Trinocular (LED) Microscpe Model: CX23 with Digital camera from Optoscient (www.optoscient.com). Ultrasound (Primo Combination 860 therapy unit purchased from S.A.G.International For Medical equipment, Nasr City, Cairo, Egypt). ChattanogaIntelect Mobile Laser 2779 Class 3B Low Level Laser Therapy (Mains Power: 120-240 VAC, $5060 \mathrm{~Hz}$ purchased from ServomedInternational Co., Cairo, Egypt, 1, Abdel WahabSelim Al-Beshryst.,Sheraton-Heliopolis).

\section{II.3Experimental animal grouping and chemical induction of unilateral $\mathrm{OA}$ in in the knee joint:}

Eighteen young adult male Albino rats weighing $(150-200 \mathrm{gm})$ purchased from the Animal Core Facility, Faculty of Agriculture, Minya University. Rats were kept in the Physiology laboratory, College of Vet. Medicine, Minya University for 1 week before start of the experimental protocol. Rats were allowed food and water ad-libitum and housed as three rats/ cage with $12 \mathrm{~h}$. light/ dark cycle at $25^{\circ} \mathrm{C}$. The experiments were carried out according to the protocol approved by the Local Ethical Committee of the Faculty of Medicine, MinyaUniversity in Accordance with the Ethical Guidelines for Scientific Research in conscious animals.

For induction of OA, all rat groups received $200 \mathrm{mg} / \mathrm{Kg}(25 \mu \mathrm{l}$ of $2 \%$ saline solution of Levox injected s.c. twice two days apart) [9].Rats divided into three groups, six animals each; (Lev) group treated with levofloxacin alone, (US) group treated with Lev plusunder water US application at frequency $=1 \mathrm{MHz}$, area of head $=3 \mathrm{~cm}^{2}$, power density $=1.5 \mathrm{~W} / \mathrm{cm}^{2}$ for $8 \mathrm{~min}$. focused on the knee joints interline with slow, circular and rhythmic movements (Fig. 2a) [10], [11] and (LLLT) groups treated with Lev plus LLLT concentrated on the right knee joint interline only at $850 \mathrm{~nm}$ (Chattanooga), power output $=200 \mathrm{~mW}$, fluence $=3.6 \mathrm{~J} / \mathrm{cm}^{2}$ and frequency $=16 \mathrm{~Hz}$. for $20 \mathrm{~s}$. [12] (Fig.1a and b).US and Laser were applied (2 to 3 times $/$ week for 4 weeks) a total of ten sessions were given.

\section{II.4 Surgical techniques:}

At the end of the experiment, all animals anaesthetized with anaesthetized with chloroform. Blood samples from experimental animals were taken from the retro-orbital plexus of veins by capillary tube and collected into glass test tubes. Serum was separated and kept at $-30^{\circ} \mathrm{C}$ for further biochemical analysis. Animals were euthanized by decapitation done by experienced Staff member. Knees of both legs from all animals were dissected from the coxofemoral joint to the ankle joint. Skin and soft tissues including the musclesremoved leaving the articular capsule intact. After the dissection, the maximum extension angle of each knee was measured [13], with zero degrees corresponding to the maximum possible extension(Fig.2d). For minimizing bias, all measurements of the extension level performed by the same surgeon.

\section{II.5 Technique of histopathology:}

Osteotomy $3 \mathrm{~cm}$ above and below the knee joint done and the knee joint with part of the femur above tibia and fibulabelow was dissected and digital photographs of the articular surfaces taken (Fig. 2 a, b \& c). Sections fixed in 10\% formaldehyde solution for 24 hours (hr.)at room temperature and decalcified in $10 \%$ nitric acid for $36 \mathrm{hr}$. Then the specimens washed in tap water and reincubated in $10 \%$ formaldehyde for $24 \mathrm{hr}$. After incubation in buffers with gradually increasing concentration of alcohol and xylol, the samples were embedded in paraffin, cut in $5 \mu \mathrm{m}$ sections and were stained with hematoxylin/eosin solution. The histological sections examined using OLYMPUS Laboratory Trinocular (LED) Microscope with Digital camera. The injuries of articular and epiphyseal cartilage plates of rats' knees measured using the Mankin score after Armstrong [14]. The higher the score, the higher the level of OA in this system. To eliminate bias, thesame histopathologist who were blinded to group distribution performed the entire histological evaluation. 


\section{II.6 Statistical methods:}

All statistical analysis completed using GraphPad Prism 6.07 (GraphPad Software Inc., La Jolla, CA, USA). All statistics presented as mean \pm one standard error of the mean. Data were compared among groups using One-Way Analysis of Variance (ANOVA) followed by NewmannKeulsposthoc test. A value of $p<0.05$ is selected to indicate statistical significance.

\section{Results \\ III.1 Effect of continuous ultrasound and low-level laser therapy on the knee extension angle in levofloxacin induced rat model of $O A$. \\ Both US and LLLT application caused significant increase in knee extension angle compared to their positive controls; treated with Lev. alone. Insignificant difference in knee extension angle between US group and LLLT group (Fig. 2e).}

III.2 Effect of continuous ultrasound and low-level laser therapy on the serum levels of interleukin1-beta, interleukin 6 and interleukin-10 in levofloxacin induced rat model of $O A$.

The results of the current study showed significant decrease in IL1-beta in both LLLT and US groups compared to Lev group. Significant decrease in IL-6 in US group compared to both Lev group and LLLT group. Highly significant increase in IL-10 in US group compared to both Lev Group and LLLT group. Insignificant difference in IL-6 and IL-10 between Lev group and LLLT group.

III.3 Effect of continuous ultrasound and low-level laser therapy on the serum levels of Tumor necrosis factor-alpha (TNF $\square$ ) and Interferon-gamma (INF- $\square$ ) in levofloxacin induced rat model of OA.

We demonstrated significant decrease in serum levels of INF-gamma in US group compared to both Lev group and LLLT group. Insignificant difference in INF-gamma between Lev group and LLLT group. Insignificant difference in TNF-alpha between the three groups was found.

\section{III.4 Effect of continuous ultrasound and low-level laser therapy on the serum level of $\mathrm{C}$ reactive protein (CRP) in levofloxacin induced rat model of $\mathrm{OA}$}

The present work showed significant decrease in CRP in US group compared to both Lev group and LLLT group. Insignificant difference in CRP between Lev group and LLLT group was obtained.

\section{III.5 Effect of continuous ultrasound and low-level laser therapy on the knee joint histology in levofloxacin induced rat model of $\mathrm{OA}$.}

Sections of rat knee joints stained with Hematoxylin and eosin showing Fig.6I (A1-4): control healthy smooth articular cartilage with normal shape, cellularity and layers (A1-2). Healthy epiphyseal cartilage plates with intact osteochondral junction showing all layers of epiphyseal cartilage plate intact (A3 \& 4). Fig.6I (B14): Sections of rat knee joints treated with Lev $200 \mathrm{mg} / \mathrm{Kg}$ showing low quality cartilage with altered cartilage structure, matrix loss, hypocellularity, onset of cell clones, surface abrasions $(*)$ and disrupted osteochondral junction (\#). Fig.6I (C1-4): Sections of rat knee joints treated with Lev and given 10 sessions of continuous therapeutic US showing good quality cartilage with smooth surface without irregularities (*), and intactosteochondral junction (\#). D1-4: Sections of rat knee joints treated with Lev and given 10 sessions of LLLT showing good quality cartilage with smooth surface without irregularities $(*)$, and intactosteochondral junction (\#).We found significant lowering of Mankin's score in both US and LLLT groups compared to Lev group (Fig.6II).

\section{Discussion}

Inflammation and the resulting elevation of proinflammatory cytokines are one of the pathophysiological characteristics of OA[15]. Both proinflammatory IL-1 $\beta$ and TNF $\alpha$ as well as antiinflammatory mediators IL-10 produced by synovial cells, chondrocytes or leukocytes are elevated in the course of the disease that affect the articular function. Previous studies showed that LLLT and US are considered as effective methods in reduction of inflammation associated with OA. Leong et al. reported that US provides a good preventive and therapeutic modality for OA and recommended the need for further research in this area for better understanding of its mechanism of action [16].LLLT also offers a good therapeutic modality of OA through regulating inflammatory cytokine expression [17]. In the current study, we compared the effect of continuous therapeutic US $(1 \mathrm{MHz})$ and LLLT $(850 \mathrm{~nm})$ on the serum level of inflammatory markers in the Levofloxacin-induced knee OA in rats.

The results of the current study showed significant decrease in the level of IL-1 beta in both US group and LLLT group compared to Lev group. We also showed insignificant change in TNF-alpha between the three groups used. In line with us, Assis et al reported decrease in IL1-beta with $50 \mathrm{~mW}$ laser therapy applied to 
surgically induced knee OA of rats [18]. Moreover, Chung et al. found decrease of IL1-beta but not TNF-alpha expression with $1 \mathrm{MHz}$ and intensity 30-200 mW continuous US applied for $10 \mathrm{~min}$. for five concecutive days in chemically-induced knee OA of rats [19]. The role of IL1-beta in amplifying the production of other cytokines such as IL-6, TNF-alpha and INF-gamma driving the production of matrix metalloproteinases inducing a cascade of destructive and inflammatory responses in osteoarthritis had been reported [20]. Up to this point, we may conclude that the ability of US and LLLT to reduce the levels of IL1-beta in knee OA may play an important role in blocking the all pathogenic cascade that causing OA progression.

Moreover, the present study demonstrated significant decrease in both IL-6 and INF-gamma in US group compared to Lev group. However, we found insignificant difference in IL-6, INF-gamma in LLLT group compared to Lev group. Supporting our results, a previous study reported the involvement IFN- $\gamma$ in the course of inflammation and cartilage damage in OA [21]. Another study by Nasonova et al. showed that anti-INF gamma was superior to anti-TNF-alpha in treatment of rheumatoid arthritis [22],[23]. Several studies demonstrated that administration of anti-interleukin 6 receptor antibody improve manifestation and prevent progression of rheumatoid arthritis [24],[25]. However, no previous study showed the direct effect of US or LLLT on INF-gamma levels in OA or the use of anti-INF-gamma in treatment of OA. In addition, we found significant elevation of IL-10 in US group compared to both Lev and LLLT groups. Insignificant difference in IL-10 in LLLT group compared to Lev. group was found. The important role of IL-10 in natural inhibition of arthritis had been reported [26].Guo et al. reported better effect of US compared to laser on knee OA of rabbits [27]. In contrast to us, Alves et al. reported decreased expression of not only IL-1 beta but also IL-6 and TNFalpha by using $50 \mathrm{~mW}$ laser therapy and higher reduction of TNF alpha with $100 \mathrm{~mW}$ in joint inflammation induced by papain [12]. Aimbire et al. reported dose dependent decrease in TNF-alpha using LLLT starting at 0.11 Joules administered with a power density of $31.3 \mathrm{~mW} / \mathrm{cm} 2$ in $42 \mathrm{sec}$ in acute lung injury caused by intravenous injection of $10 \mathrm{mg}$ of ovalbumin in $0.5 \mathrm{~mL}$. This apparent conflict may be explained on the basis of different animal model of OA used, or different parameters of LLLT used or number and duration of therapeutic sessions given [28].

Previous studies showed that the elevation of serum CRP is associated with knee as well as hip osteoarthritis prediction and severity if compared to healthy individuals [29],[30]. Our results demonstrated significant decrease in CRP in US group compared to Lev group and LLLT group. Insignificant change in CRP was found in LLLT group compared to Lev group. Up to our knowledge, no previous literature showed the effect of conventional US or LLLT on the serum level of CRP in OA of the knee joint. One study reported decrease in post-exercise elevation of blood CRP as well as lactate and creatine kinase due to pre-exercise irradiation by low-level laser [31].According to the results of the current study, continuous US therapy was more effective in reduction of the inflammatory markers; IL-6, INF-gamma as well as elevation of the antiinflammatory cytokine IL-10 in the serum of Lev-induced OA of knee joint in rats.

In order to further prove the higher efficacy of US compared LLLT, we compared the histopathologicchanges in knee joint of rats treated with Lev alone and rats treated with Lev+USor Lev+ LLLT using Mankin Score. Histopathological examination of the knee joints of Lev group showed marked damage of epiphyseal cartilage plate as well as the articular cartilage. We found matrix loss, hypocellularity, cell clones, surface abrasions and disrupted osteochondral junctions. Therefore, our results further support the use of Lev to develop a good model of OA. Although both US and LLLT were fairly good treatment of knee OA as evidenced by histopathology, US was more effective in returning the knee joint to its normal architecture.However, both treatment produced significantly lower score compared to Lev. group. In line with us, Brosseau et al reported that although LLLT was recommended for relief of pain and stiffness in rheumatoid arthritis (RA), it gives contradictory results in OA pending on wavelength, dosage, duration and site of application [32]. They recommend further studies to detect the previously mentioned parameters for both RA and OA. Moreover, Angelova and Ilieva demonstrated that high intensity laser (HILT) produced intense and cumulative effect in comparison to LLLT after seven sessions of treatment [33]. They preferred the use HILT as a method of choice in the treatment of the pathological changes involving damage of articular cartilage and improving the reactive changes in both synovial membrane and synovial fluid.Although both US and LLLT were effective in lowering IL-1 beta, limitation in knee extension and Mankin's score of knee histopathology, US was more effective in lowering theproinflammatory cytokines IL-6, INF-gamma, CRP and significantlyelevatingthe anti-inflammatory cytokine IL-10. Taken together, we may speculate that US was more effective in treatment of Lev-induced knee $\mathrm{OA}$ in rats. We recommend the use of US for treatment of OA and strongly encourage further studies in both animals and humans to investigate the role of HILT for management of cases of OA. 


\section{Conclusion}

In the present work, we used Levofloxacin to develop rat model of knee osteoarthritis. We divided the animals into three groups; positive control treated with Lev. only, US group treated with both Lev. and US (1 MHz), LLLT group treated with Lev. and exposed to LLLT $(850 \mathrm{~nm})$. The results of our study showed that US gave better results in treatment of knee OA in rats.UScaused significant decrease in proinflammatory cytokines IL-6, INF-gamma, CRP and rise of anti-inflammatory cytokines IL-10 compared to both Lev group and LLLT group. However, both US and LLLT caused significant increase in knee extension angle and decrease in IL-1 beta as well as lower Mankin's score. Therefore, we recommend the use of US for treatment of OA.Further studies are needed to investigate the role of HILT for management of cases of OA.

\section{References}

[1]. M. Attur, S. Krasnokutsky-Samuels, J. Samuels, S.B. Abramson. Prognostic biomarkers in osteoarthritis. CurrOpinRheumatol. 25(1), 2013, 136-44

[2]. P. Wojdasiewicz, L.A. Poniatowski, D. Szukiewicz. The role of inflammatory and anti-inflammatory cytokines in the pathogenesis of osteoarthritis. Mediators of inflammation.Vol 2014, article ID 561459, 19 pages.

[3]. K. Lampropoulou-Adamidou, P. Lelovas, E.V. Karadimas, C. Liakou, I.K. Triantafillopoulos, I. Dontas, et al. Useful animal models for the research of osteoarthritis. Eur J OrthopSurgTraumatol. 24(3), 2014, 263-71.

[4]. R.W. Moskowitz, R.D. Altman, M.C. Hochberg, J.A. Buckwalter, V.M. Goldberg. Experimental models of osteoarthritis. In: Moskowitz RW, Goldberg VM, Hochberg MC, editors. Osteoarthritis: diagnosis and medical/surgical management. 4th ed. Lippincott Williams \& Wilkins; 2007:107-25.

[5]. C. Zeng, H. Li, T. Yang, Z.H. Deng, Y. Yang, Y. Zhang, X. Ding, G.H. Lei. Effectiveness of continuous and pulsed ultrasound for the management of knee osteoarthritis: a systematic review and network meta-analysis. Osteoarthritis Cartilage. 22(8), 2014, 10909 .

[6]. S. Cakir, S. Hepguler, C. Ozturk, M. Korkmaz, B. Isleten, F.C. Atamaz. Efficacy of therapeutic ultrasound for the management of knee osteoarthritis: a randomized, controlled, and double-blind study. Am J Phys Med Rehabil. 93(5), 2014, 405-12.

[7]. B. Hegedüs, L. Viharos, M. Gervain, M. Gálfi. The effect of low-level laser in knee osteoarthritis: A Double-Blind, Randomized, Placebo-Controlled Trial. Photomed Laser Surg. 27(4), 2009, 577-84.

[8]. E.F. Youssef, Q.I. Muaidi, A.A. Shanb. Effect of laser therapy on chronic osteoarthritis of the knee in older subjects. J Lasers Med Sciv.7(2); Spring 2016PMC4909009.

[9]. A.W. Gough, O.B. Kasali, R.E. Sigler, V. Baragi. Quinolone arthropathy--acute toxicity to immature articular cartilage. ToxicolPathol. 20(3 Pt 1), 1992, 436-49; discussion 449-50.

[10]. D.G. Külcü, G. Gülșen, E.C. Altunok. Short-term efficacy of pulsed electromagnetic field therapy on pain and functional level in knee osteoarthritis: A randomized controlled study. Turk J Rheumatol 24, 2009, 144-8.

[11]. J.G. Coradini, T.F. Mattjie, G.R. Bernardino, A.L. Peretti, C.M.M. Kakihata, T.K. Errero, A.R. Escher, G.R.F. Bertolini. Comparison of low level laser, ultrasonic therapy and association in joint pain in Wistar rats. Rev. Bras. Reumatol 54(1), 2014, 712.

[12]. A.C. Alves, R. Vieira, E. Leal-Junior, S. dos Santos, A.P. Ligeiro, R. Albertini, J. Junior, P. de Carvalho. Arthritis Res Ther. 15(5), 2013, R116.

[13]. M.U. de Rezende, HM de Campos Gurgel, P.R. Vilaça Junior, R.K. Kuroba, A.S.S. Lopes, R.Z. Phillipi, et al. Diacerein versus glucosamine in a rat model of osteoarthritis. CLINICS 61(5), 2006, 461-6.

[14]. S. Armstrong, R. Read, P. Ghosh. The effects of intraarticularhyaluronan on cartilage and subchondral bone changes in an ovine model of early osteoarthritis. J Rheumatol. 21, 1994, 680-8.

[15]. M.J. Benito, D.J. Veale, O. FitzGerald, W.B. van den Berg, B. Bresnihan. Synovial tissue inflammation in early and late osteoarthritis. Ann Rheum Dis 64(9), 2005, 1263-7.

[16]. D.J. Leong, H. Zhang, L. Xu, J. Tang, D.M. Hirsh, et al. Therapeutic ultrasound: osteoarthritis symptom-modification and potential for disease modification. J Surgery. 1(2), 2013, 5.

[17]. R.C. Pallotta, J.M. Bjordal, L. Frigo, E.C. Leal Junior, S. Teixeira, R.L. Marcos, L. Ramos, F. de M Messias, R.A. Lopes-Martins. Infrared (810-nm) low-level laser therapy on rat experimental knee inflammation. Lasers Med Sci. 27(1), 2012, 71-8.

[18]. L. Assis, L.P. Milares, T. Almeida, C. Tim, A. Magri, C. Fernandes, C. Medalha, A.C. Renno. Aerobic exercise training and lowlevel laser therapy modulate inflammatory response and degenerative process in an experimental model of knee osteoarthritis in rats. Osteoarthritis and Cartilage. 24(1), 2016, 169-77.

[19]. J.I. Chung, S. Barua, B.H. Choi, B.H. Min, H.C. Han, E.I. Baik. Anti-inflammatory effect of low intensity ultrasound (LIUS) on complete Freund's adjuvant-induced arthritis synovium. (20)4, 2012, 314-22.

[20]. A. Melo-Florián. IL-1 and its role in osteoarthritis. Open Journal of Medicine. 1(1), 2011, 3.

[21]. H. Ishii, H. Tanaka, K. Katoh, H. Nakamura, M. Nagashima, S. Yoshino. Characterization of infiltrating T cells and Th1/Th2-type cytokines in the synovium of patients with osteoarthritis. Osteoarthritis and Cartilage. 10(4), 2002, 277-81.

[22]. V.A. Nasonova, G.V. Lukina, Ia.A. Sigidin. Neutralisation of interferon gamma--a new trend in therapy of rheumatoid arthritis. TerArkh. 80(5), 2008, 30-7.

[23]. Y.A. Sigidin, G.V. Loukina, B. Skurkovich, S. Skurkovich. Randomized, double-blind trial of anti-interferon-gamma antibodies in rheumatoid arthritis. Scand J Rheumatol. 30(4), 2001, 203-7.

[24]. J.S. Smolen, A. Beaulieu, A. Rubbert-Roth, C. Ramos-Remus, J. Rovensky, E. Alecock, et al. Effect of interleukin-6 receptor inhibition with tocilizumab in patients with rheumatoid arthritis (OPTION study): a double-blind, placebo-controlled, randomised trial. Lancet. 371(9617), 2008, 987-97.

[25]. R.N. Maini, P.C. Taylor, J. Szechinski, K. Pavelka, J. Broll, G. Balint, et al. Double-blind randomized controlled clinical trial of the interleukin-6 receptor antagonist, tocilizumab, in European patients with rheumatoid arthritis who had an incomplete response to methotrexate. Arthritis Rheum. 54(9), 2006, 2817-29.

[26]. I.C. Helmark, U.R. Mikkelsen, J. Børglum, A. Rothe, M.C.H. Petersen, O. Andersen, H. Henning Langberg, M. Kjaer. Exercise increases interleukin-10 levels both intraarticularly and peri-synovially in patients with knee osteoarthritis: a randomized controlled trial. Arthritis Research \& Therap. 12, 2010, R126.

[27]. H. Guo, Q. Luo, J. Zhang, H. Lin, L. Xia, C. He. Comparing different physical factors on serum TNF- $\alpha$ levels, chondrocyte apoptosis, caspase-3 and caspase- 8 expression in osteoarthritis of the knee in rabbits. Joint Bone Spine. 78(6), 2011, 604-10. 
[28]. F. Aimbire, R. Albertini, M.T.T. Pacheco, H.C. Castro-Faria-Neto, P.S.L.M. Leonardo, V.V. Iversen, Dr.R.A.B. Lopes-Martins, J.M. Bjordal. Low-level laser therapy induces dose-dependent reduction of TNF $\alpha$ levels in acute inflammation. Photomedicine and Laser Surgery. 24(1), 2006, 33-37.

[29]. J.W. Smith, T.B. Martins, E. Gopez, T. Johnson, H.R. Hill, T.D. Rosenberg. Significance of C-reactive protein in osteoarthritis and total knee arthroplasty outcomes. Therapeutic Advances in Musculoskeletal Disease. TherAdvMusculoskel Dis. 4(5), 2012, 315-25.

[30]. A.D. Pearle,C.R. Scanzello, S.S. George, L. Mandl, E.F. DiCarlo, M.K. Crow, T.P. Sculco1. Elevated C-reactive protein levels in osteoarthritis are associated with local joint inflammation. Arthritis Res Ther. 6(3), 2004, 56.

[31]. E.C.P. Leal, R.Á.B. Lopes-Martins, L. Frigo, T.D. Marchi, R.P. Rossi, V.D. Godoi, et al. Effects of low-level laser therapy (LLLT) in the development of exercise-induced skeletal muscle fatigue and changes in biochemical markers related to postexercise recovery. Journal of Orthopaedic and Sports Physical Therapy. 40(8), 2010, 524-32.

[32]. L. Brosseau, V. Welch, G. Wells, P. Tugwell, R. de Bie, A. Gam, K. Harman, B. Shea, M. Morin. Low level laser therapy for osteoarthritis and rheumatoid arthritis: a meta-analysis. J Rheumatol. 27(8), 2000,1961-9.

[33]. A. Angelova,E.M. Ilieva. Effectiveness of high intensity laser therapy for reduction of pain in knee osteoarthritis. Pain Research and Management. 2016(2016), Article ID 9163618, 2016, 11 pages.

\section{Figures}

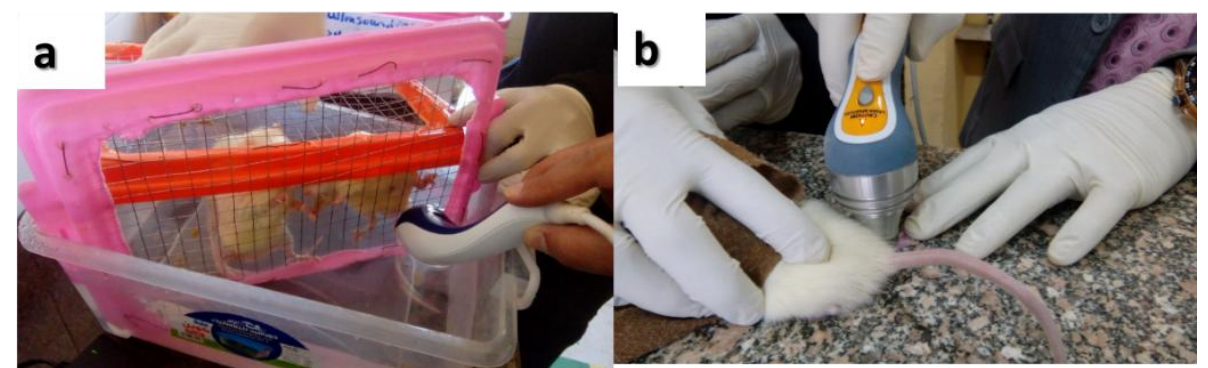

Fig 1: Method of application of Ultrasound (US) and Low level laser (LLLT).

Application of continuous ultrasound (a), application of low level laser (b).
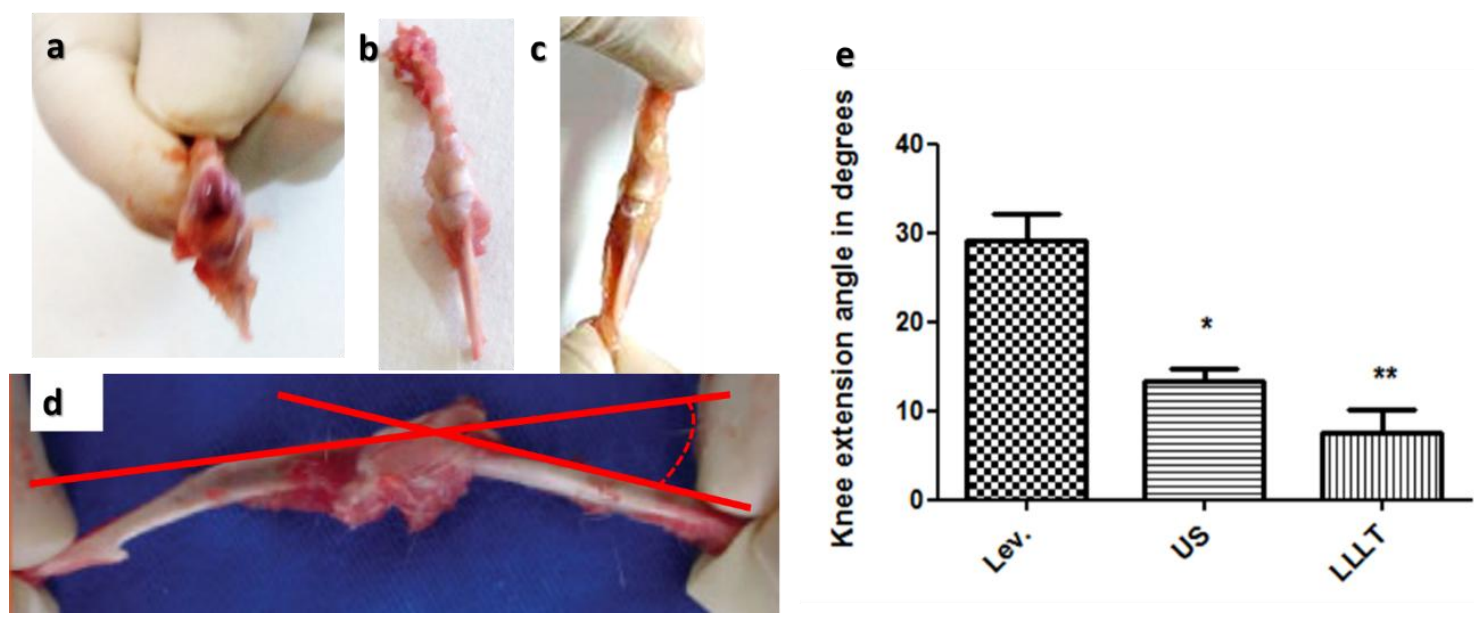

Fig 2: Effect of continuous ultrasound and low-level laser therapy on the knee extension angle in levofloxacininduced rat model of OA. (a) an image to of knee of Lev group; (b) an image of healthy knee from the US group; (c) an image of knee from LLLT group; (d) measurement of knee extension angle as zero degrees considered as maximum extension; (e) comparison of knee extension angle in Lev: levofloxacin group, US: ultrasound group, LLLT: low level laser group, OA: osteoarthritis, data represents mean \pm SD; (*) US group or LLLT group vs Lev group, ${ }^{*} \mathrm{P}<0.05,{ }^{* *} \mathrm{P}<0.01, \mathrm{~N} .=4$ in $\mathrm{C}$ group, $\mathrm{N} .=10$ legs in Lev and US group, N. $=6$ in LLLT group. 




Fig 3: Effect of continuous ultrasound and low-level laser therapy on the serum levels of IL1 $\beta$ (interleukin1beta), IL-6 (interleukin 6) and IL-10 (interleukin-10) in levofloxacin induced rat model of OA. Lev: levofloxacin group, US: ultrasound group, LLLT: low-level laser group; data represents mean \pm SD; $(*)$ US group or LLLT group vs Lev group, (+) US group vs LLLT group, ${ }^{* * *} \mathrm{P}<0.005,{ }^{*} \mathrm{P}<0.05,{ }^{++} \mathrm{P}<0.001, \mathrm{~N}$. $=6$ in each group.

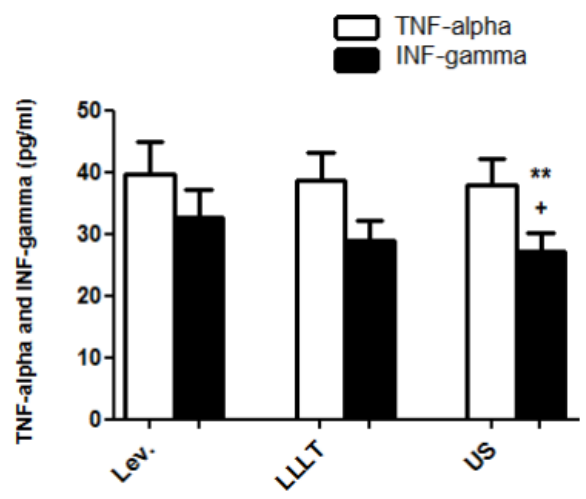

Fig 4 Effect of continuous ultrasound and low-level laser therapy on the serum levels of TNF $\alpha$ (Tumor necrosis factor-alpha) and INF- $\gamma$ (Interferon-gamma) in levofloxacin induced rat model of OA. Lev: levofloxacin group, US: ultrasound group, LLLT: low-level laser group; data represents mean \pm SD; (*) US group or LLLT group vs Lev group, (+) US group vs LLLT group, ${ }^{* *} \mathrm{P}<0.01,{ }^{+} \mathrm{P}<0.05, \mathrm{~N} .=6$ in each group.

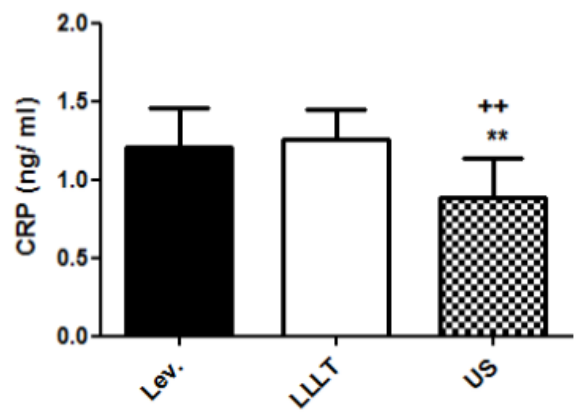

Fig 5: Effect of continuous ultrasound and low level laser therapy on the serum levels of $\mathrm{C}$ reactive protein (CRP) in levofloxacin induced rat model of OA. Lev: levofloxacin group, US: ultrasound group, LLLT: low level laser

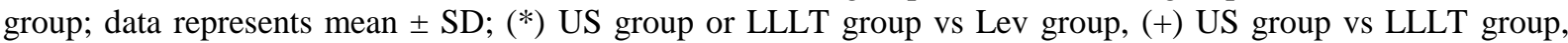
$* * \mathrm{P}<0.01,++\mathrm{P}<0.01, \mathrm{~N} .=6$ in each group. 

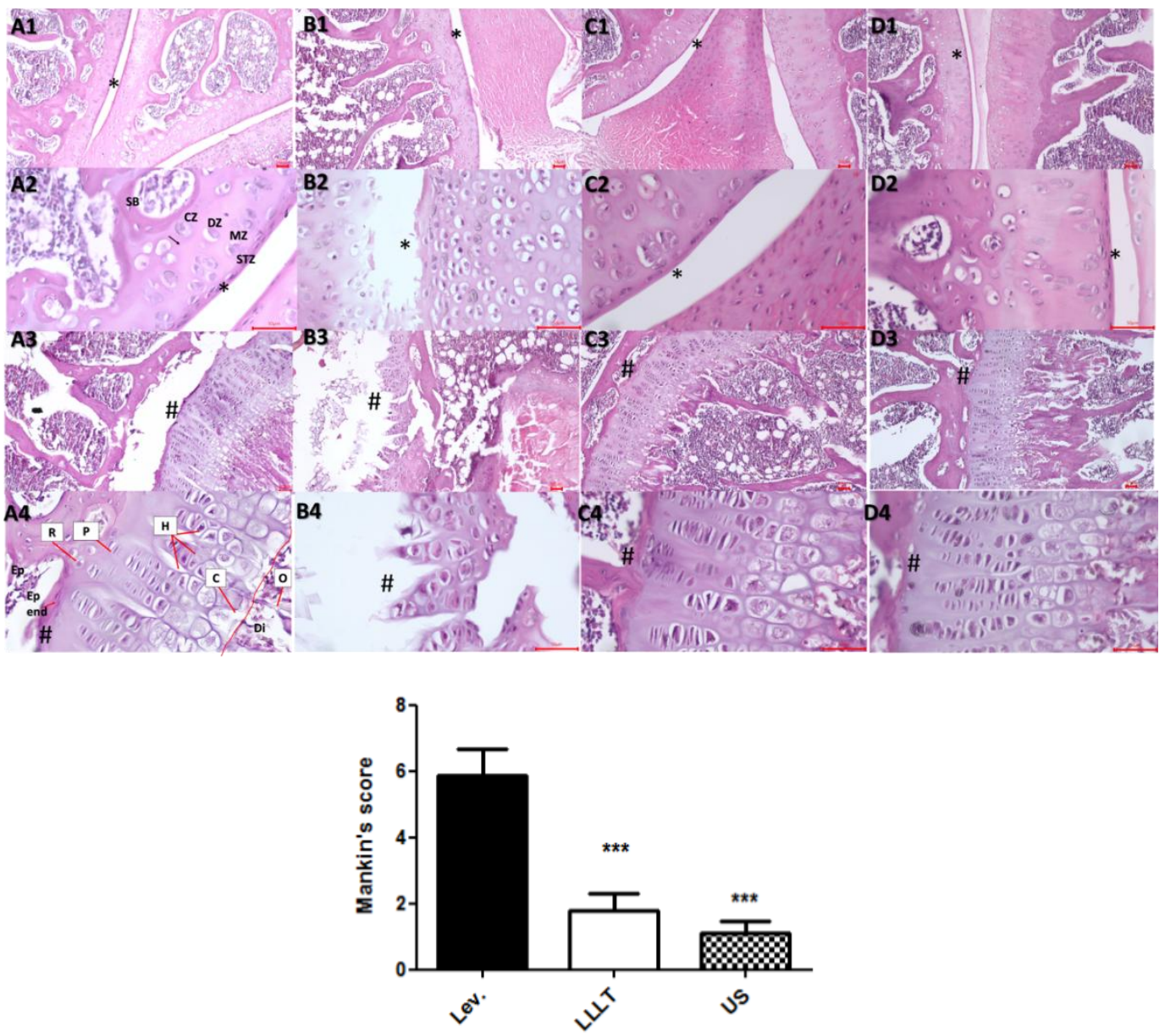

Fig 6: Effect of continuous ultrasound and low level laser therapy on the knee joint histopathology in levofloxacin induced rat model of OA. IA: Good quality cartilage of 3 month old healthy male albino rat showing smooth surface without irregularities (*), details of the normal cellular organization of the articular cartilage; STZ: superficial zone, MZ: middle zone, DZ: deep zone, CZ: calcified zone, SB: subchondral bone and showing the presence of the tidemark (black arrow); the osteochondral junction is intact (\#) showing all layers of epiphyseal cartilage plate A4; Ep: epiphysis, Di: diaphysis; R: reserve zone, P: proliferation zone, H: hypertrophied zone, $\mathrm{C}$ calcification zone and $\mathrm{O}$ : ossification zone. IB: low quality cartilage of 3 month old male albino rat treated with levofloxacin $200 \mathrm{mg} / \mathrm{Kg}$ showing altered cartilage structure, matrix loss, hypocellularity, onset of cell clones, surface abrasions $(*)$ and disrupted osteochondral junction (\#). IC: Good quality cartilage of 3 month old male albino rat treated with levofloxacin $200 \mathrm{mg} / \mathrm{Kg}$ and 10 sessions of continuous therapeutic ultrasound showing smooth surface without irregularities $(*)$, and the osteochondral junction is intact (\#). D: Good quality cartilage of 3 month old male albino rat treated with levofloxacin $200 \mathrm{mg} / \mathrm{Kg}$ and 10 sessions of low level laser therapy showing smooth surface without irregularities $\left(^{*}\right)$, and the osteochondral junction is

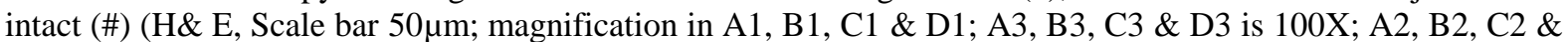
D2; A4, B4, C4 \& D4 400X). II: Graph showing comparison of Mankin's score in Lev: levofloxacin group compared to US: ultrasound group and LLLT: low level laser therapy group (*) US group or LLLT group vs Lev group, $* * * \mathrm{P}<0.005, \mathrm{~N} .=6$ in each group. 